\title{
NOUVELL
}

\section{Les cellules lymphoïdes \\ innées contrôlent la réponse adaptative aux bactéries commensales intestinales}

Natalia Korneychuk
Inserm U989, Université Paris Descartes-Sorbonne Paris Cité, Institut Imagine, 96, rue Didot, 75014 Paris, France. natkorneychuk@yahoo.fr
> Le tube digestif est colonisé par environ 100 trillions de bactéries composées de milliers de souches différentes [1, 11] $(\rightarrow)$. Chez un sujet sain, la flore

$\rightarrow$ Voir la synthèse de A. El Kaoutari et al., microbienne est page 259 de ce numéro
confinée dans des endroits précis et maintient des relations symbiontes avec I'hôte. La flore assure non seulement des processus physiologiques normaux, mais aussi le développement du système immunitaire de l'hôte, tout en limitant la colonisation par des microbes potentiellement pathogènes (Figure 1). Les modifications de la composition et/ou de la localisation anatomique des bactéries intestinales sont associées à de multiples maladies infectieuses, inflammatoires ou métaboliques (hépatite virale, infection par le VIH, maladies inflammatoires chroniques intestinales, cancer, diabète, obésité, maladies cardiovasculaires) [2] (Figure 1). Un lien étroit a été établi entre la flore intestinale et les deux composantes du système immunitaire - immunité adaptative et immunité innée. Un nouveau groupe de cellules lymphoïdes innées (ILC, innate lymphoid cells) semble jouer un rôle important dans ces interactions.

\section{Les cellules lymphoïdes innées}

Les cellules lymphoïdes innées sont une population nouvellement décrite de cellules immunitaires innées qui partagent de nombreuses caractéristiques développementales et fonctionnelles avec les

N.K. bénéficie d'une bourse de l'Université Paris Descartes pour une mission doctorale à médecine/sciences. lymphocytes T CD4 ${ }^{+}$ (Figure 2) [3] $(\rightarrow$ ). $(\rightarrow)$ Voir la Synthèse de Marie Cherrier, page $\mathbf{2 8 0}$ de ce numéro flammation et le confinement anatomique de bactéries pathogènes et commensales dans l'intestin [5-7]. Les LTi, nécessaires au développement de ganglions et de plaques de Peyer, sont une des sous-populations les plus connues des ILC de groupe 3. De plus, les ILC ROR $\gamma \mathrm{t}^{+}$sont les premières cellules du système immunitaire à coloniser l'intestin néonatal et les tissus associés. Récemment, Sonnenberg et al. ont mis en évidence un rôle important des ILC de groupe 3 dans la régulation de la réponse adaptative $\mathrm{T} C D 4^{+}$aux bactéries commensales [8]. L'étude effectuée par Hepworth et al., que nous discutons ici, complète ces données [9].

dans les régions barrières du corps $d$ mammifères, la peau, les voies respiratoires et le tube intestinal [4]. Étant donné la proximité spatiale entre les bactéries, les cellules épithéliales et les ILC, plusieurs groupes ont étudié l'effet de bactéries commensales sur le développement des ILC. Les signaux émis par les bactéries commensales semblent réguler la fonction des ILC soit directement - via les récepteurs TLR (toll-like receptor), AhR (aryl hydrocarbon receptor), NCR (natural cytotoxicity receptor), soit indirectement - via la production de cytokines par d'autres populations cellulaires.

Les ILC de groupe 3 sont particulièrement impliquées dans la réponse intestinale. Ce groupe inclut toutes les ILC dont le développement et la fonction dépendent du facteur de transcription ROR $\gamma$ t et qui, en réponse à la stimulation par I'IL-23, produisent de I'IL-17A et/ou de I'IL-22 (Figure 2). Ces cytokines sont impliquées dans l'induction de l'immunité innée, l'in-

\section{Étude des cellules lymphoïdes innées}

\section{de groupe 3}

Il est difficile d'analyser les ILC en raison de l'absence de marqueurs permettant de les cibler sélectivement. Chez les animaux immunocompétents, les cellules $\mathrm{T} \mathrm{CD4}^{+}$, qui expriment des profils de cytokines similaires à celui des ILC, sont dominants et masquent la fonction de ces dernières. Pour étudier le rôle des ILC de groupe 3 sans éliminer toute l'immunité adaptative, Hepworth et al. ont utilisé dans un premier temps des souris déficientes en ROR $\gamma t$ (Rorc ${ }^{g f p / g f p}$ ) [9]. Ces souris présentent plusieurs signes de rupture de l'homéostasie immunitaire : prolifération accrue de cellules $\mathrm{T} C D 4^{+}$périphériques effectrices, développement d'une splénomégalie et une production accrue d'IgG spécifiques des bactéries commensales dans le sérum, tous signes du franchissement par les bactéries de la barrière épithéliale. L'administration orale d'antibiotiques à ces souris 


\section{A Condition physiologique}

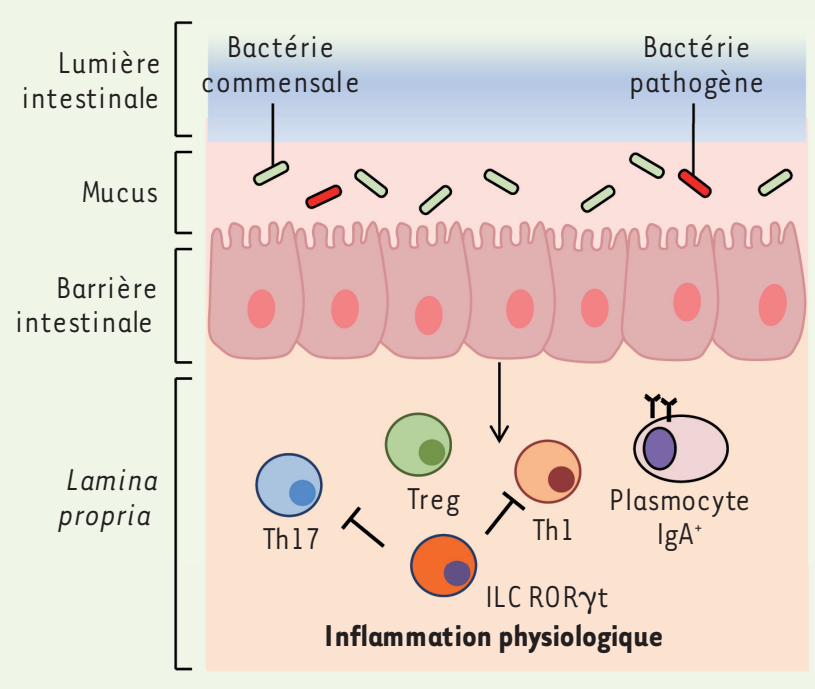

B Condition pathologique :

Antibiotiques, régime alimentaire, infections, polluants, stress

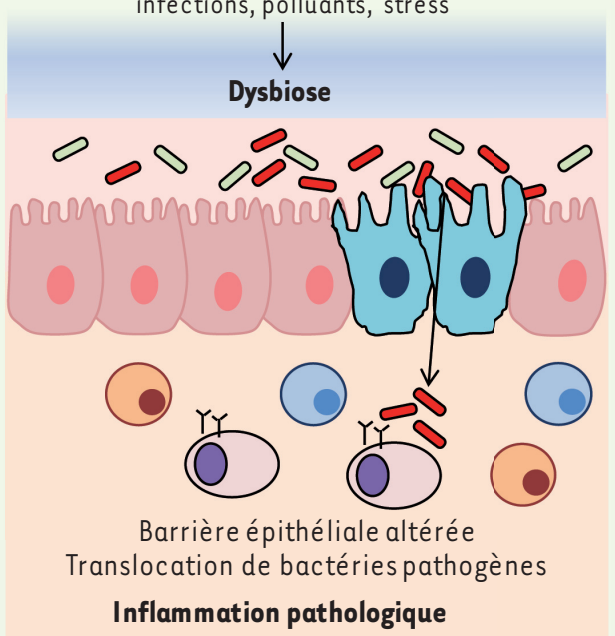

Figure 1. Présentation schématique des interactions hôte-microbiote dans l'intestin. A. Dans les conditions physiologiques, le système immunitaire contrôle le microbiote de la lumière intestinale et les mécanismes rétroactifs servent à éviter une activation excessive de la réponse immunitaire de I'hôte. Les bactéries commensales produisent des molécules anti-inflammatoires qui induisent la réponse tolérigène de l'hôte. $B$. Dans les conditions pathologiques chez des sujets immunodéficients (qui ont par exemple un défaut d'un des composants de la barrière intestinale ou d'un intermédiaire d'une des voies de signalisation impliquées), les changements de style de vie (stress, régime alimentaire, hygiène, tabac, antibiotiques, vaccins, etc.) peuvent induire une inflammation intestinale affectant l'équilibre entre le système immun de l'hôte et le microbiote intestinal. L'inflammation intestinale se traduit par une adhérence bactérienne accrue, des lésions épithéliales et la translocation accrue des bactéries dans la lamina propria. De plus, l'inflammation peut favoriser la sélection des bactéries pathogènes agressives qui sont plus résistantes aux effets des molécules antimicrobiennes de I'hôte. Ces bactéries peuvent induire la maladie même chez des sujets immunocompétents. Th : Thelper; Treg : cellule T régulatrice.

corrige de façon importante ces anomalies, suggérant que les ILC contrôlent la réponse adaptative aux antigènes bactériens. Pour éviter le biais dû à l'altération du développement des cellules T qu'entraîne l'absence de RORyt, des chimères ont été créées: des cellules $T$ et $B$ de souris C57BL/6 exprimant l'allèle CD90.1 ont été injectées à des souris congéniques (portant un autre allèle de la molécule CD90 permettant de distinguer les cellules de donneur et receveur) CD90.2 $\mathrm{Ragl}^{-/}$, donc dépourvues de lymphocytes $T$ et $B$ (Figure 3A). La déplétion des ILC CD90.2 par un anticorps monoclonal anti-CD90.2 entraîne les mêmes anomalies que l'absence de RORyt, suggérant à nouveau le rôle critique des ILC dans la régulation de la réponse immune adaptative. Celles-ci agissent indépendamment des cytokines effectrices IL-22, IL-17A, IL-23 impliquées dans la régulation et le confinement anatomique des bactéries, dont l'absence (chez des souris déficientes ou traitées par des anticorps anti-cytokines) ne modifie pas la réponse $T C D 4^{+}$.

Les ILC de groupe 3 présentent l'antigène Pour identifier les mécanismes utilisés par les ILC ROR $\gamma \mathrm{t}^{+}$de groupe 3 dans la régulation de la réponse adaptative contre la flore commensale, le profil transcriptomique des ILC ROR $\gamma \mathrm{t}^{+}$a été comparé à celui des cellules T CD $4^{+}$. Parmi les gènes fortement exprimés par les premières, on trouve ceux qui sont impliqués dans le développement des organes hématopoïétiques et lymphoïdes, dans la réponse immune et plus particulièrement dans l'apprêtement de l'antigène et la présentation des peptides via les molécules du complexe majeur d'histocompatibilité de classe II (CMH-II). Ainsi, les ILC de groupe 3 , beaucoup plus enrichies en transcrits comme Cd74, H2-DMb2, H2-DMa,
H2-Abl, H2-Aa que les cellules T CD4 ${ }^{+}$ naïves et que les Th17 générées in vitro, expriment les molécules de CMH-II ; c'est le cas des ILC de ganglions mésentériques de souris naïves ROR $\gamma$ t-eGFP comme des ILC de l'intestin grêle humain d'individus sains.

Pour prouver que les ILC sont capables, comme les cellules dendritiques, de capter et dégrader des antigènes, les ILC triées par cytométrie de flux ont été mises en contact avec un antigène fluorescent (DQ2-ovalbumine ou $\varepsilon \alpha$-GFP). L'augmentation importante de l'intensité de fluorescence de cellules ILC CMH- $\mathrm{II}^{+} \mathrm{a}$ confirmé cette fonction. Mais, contrairement aux cellules dendritiques, les ILC n'induisent pas la prolifération de cellules T CD4 $4^{+}$. On peut l'expliquer par l'absence d'expression des molécules de costimulation CD80, CD86, CD40 par les ILC. On sait que la présentation d'antigènes en l'absence de signaux de costimulation 


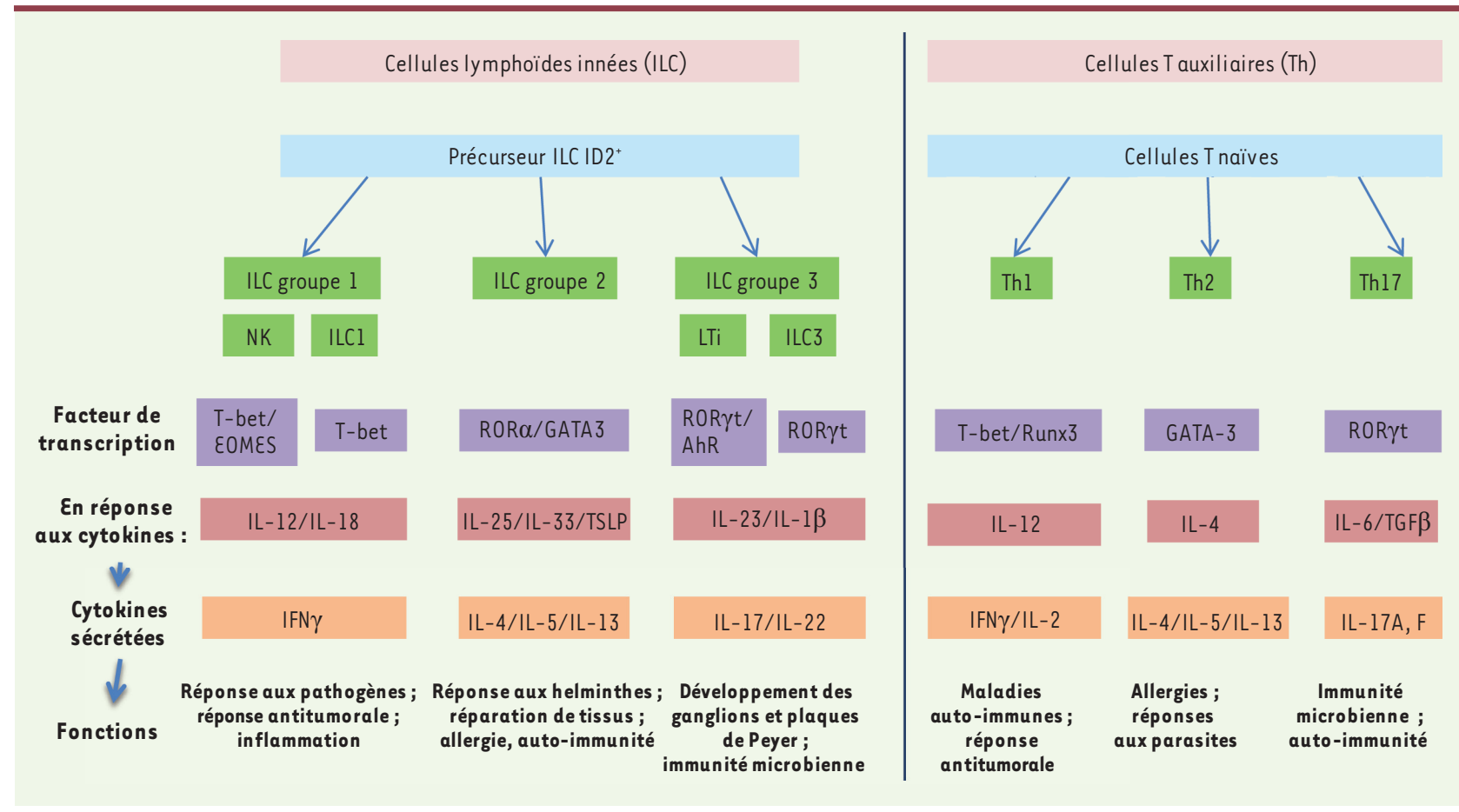

Figure 2. Classification des cellules lymphoïdes innées (ILC, innate lymphoid cells) en trois groupes sur la base de leurs caractéristiques fonctionnelles par analogie avec les cellules T auxiliaires. En fonction des cytokines sécrétées, les ILC sont séparées en trois groupes. Les ILC de groupe l sont définies par leur capacité à produire de l'IFN (comme le font les lymphocytes Thl). Les ILC de groupe 2 sont capables de produire les cytokines associées aux cellules Th2 : IL-4, IL-5 et IL-13. Les ILC de groupe 3 sont capables de produire les cytokines associées aux cellules Th17 comme l'IL-17 et l'IL-22. II est suggéré que toutes les cellules lymphoïdes innées sont issues d'un précurseur commun non identifié, exprimant Id2. Plusieurs facteurs de transcription régulent la différenciation des ILC en plusieurs sous-types. T-bet et EOMES sont les facteurs de transcription nécessaires au développement de cellules NK (incluses dans le groupe 1) [3], tandis que pour d'autres cellules ILC du groupe l, les conditions nécessaires ne sont pas complètement connues. Le développement des ILC de groupe 2 dépend des facteurs de transcription GATA-3 et ROR $\alpha$ (receptor-related orphan receptor- $\alpha$ ). En revanche, les ILC du groupe 3 ont besoin du facteur de transcription ROR $\gamma$ t pour leur développement et leur fonction. Les ILCl produisent de l'IFN $\gamma$ en réponse à la stimulation par la cytokine IL-12. La costimulation par l'IL-18 augmente significativement leur production de cytokines. Les cytokines IL-25, IL-33 et TSLP (thymic stromal lymphopoietin) induisent l'activation des ILC2 et la production des cytokines IL-4, IL-5 et IL-13. Suite à la stimulation par I'IL-23 et IL-1 $\beta$, les ILC3 sont capables de produire des cytokines IL-17 et IL-22 (d'après la classification proposée dans [9]).

induit l'anergie de cellules T. II est donc plausible que les ILC CMH-II+ RORyt modulent la réponse $\mathrm{TCD}^{+}$in vivo. De fait, la co-injection d'ILC et de T CD4 ${ }^{+}$ spécifiques de l'antigène CBirl dans les souris immunisées avec le peptide CBirl a un effet limitant sur l'expansion et la production des cytokines par les cellules T CD4 ${ }^{+}$(Figure 3B).

\section{Rôle du CMH de classe II dans la limitation par les ILC de l'activité des lymphocytes T CD4 ${ }^{+}$}

Pour étudier le rôle de $\mathrm{CMH}-\mathrm{II}$, les auteurs ont utilisé des souris dont on a éliminé de façon conditionnelle les molécules $\mathrm{CMH}-$ II spécifiquement dans les ILC ROR $\gamma t^{+}\left(\mathrm{CMH}-\mathrm{II}^{\Delta I L C}\right)$. Elles ont été obtenues en croisant des souris $\mathrm{H}_{2}-\mathrm{AbI}^{\text {fl }}$ avec des souris RorcCre (Figure $3 \mathrm{C}$ ). Cette méthode permet d'éliminer sélectivement le gène CMH-II dans les cellules ILC ROR $\gamma \mathrm{t}^{+}$, tout en préservant l'immunité adaptative et innée puisque l'expression du CMH-II sur les cellules B, dendritiques et macrophages reste intacte. Le phénotype de ces souris était identique à celui de souris déficientes en ILC. De plus, ces anomalies étaient facilement corrigées par l'administration d'antibiotiques. Ces données suggèrent que c'est via le processus de présentation d'antigène que les cellules ILC CMH- $\mathrm{II}^{+}$modulent la réponse $\mathrm{TCD}^{+}$aux antigènes issus des bactéries commensales.

L'analyse approfondie de souris $\mathrm{CMH}-\mathrm{II}^{\mathrm{\Delta LC}}$ a révélé qu'en présence d'ILC dépourvues de $\mathrm{CMH}-\mathrm{II}$, la réponse $\mathrm{TCD} 4^{+}$est exacerbée et s'accompagne d'une forte production des cytokines pro-inflammatoires IFN $\gamma$ (interféron), IL-17A, TNF $\alpha$ (tumor necrosis factor), déclenchant l'inflammation observée dans le côlon de ces souris. L'administration d'antibiotiques abolissait ce processus inflammatoire. Ces résultats suggèrent que les ILC limitent la réponse $T C D 4^{+}$spécifique de bactéries commensales par un mécanisme faisant intervenir les molécules CMH-II. 


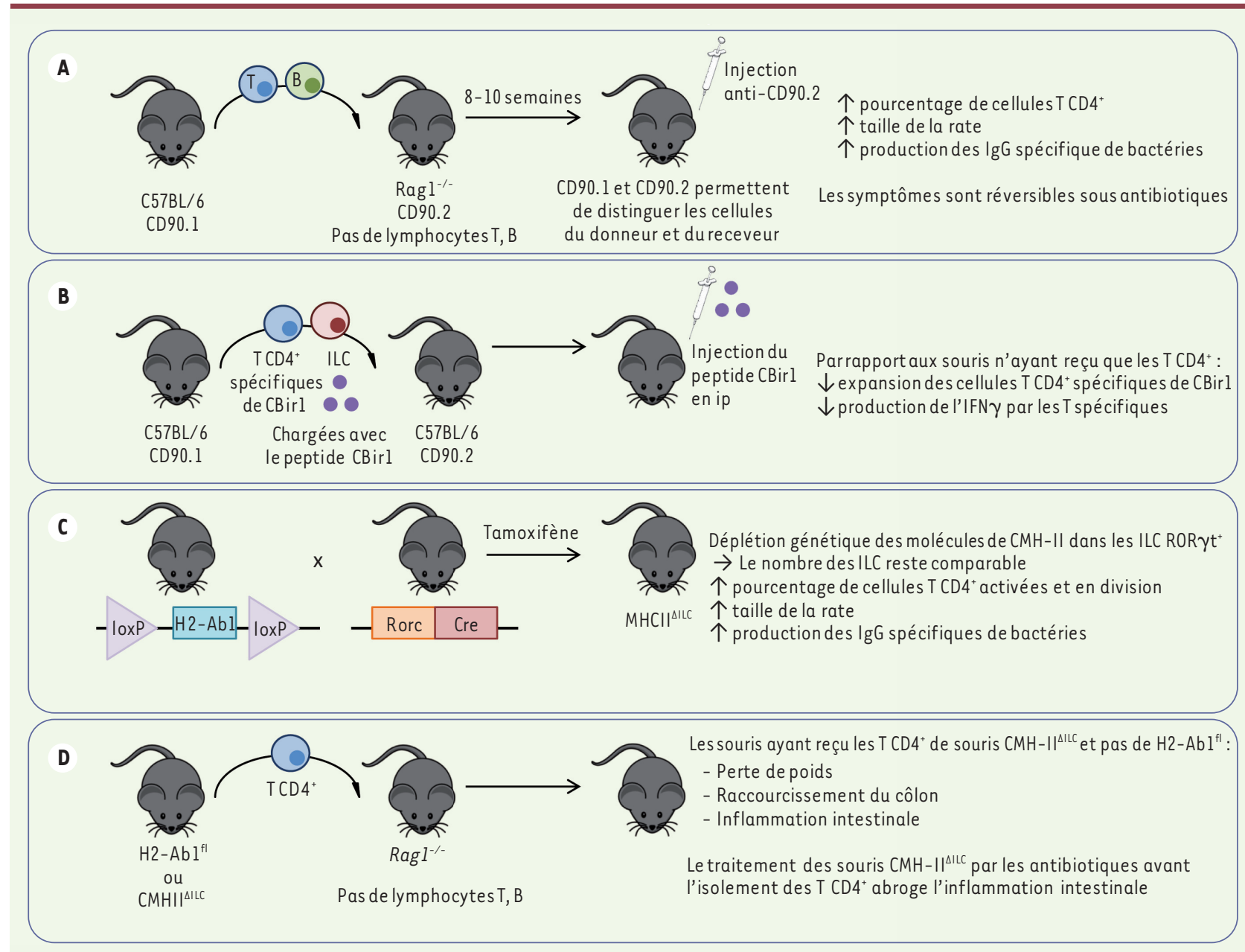

Figure 3. Résumé des stratégies expérimentales utilisées. Démonstration du rôle des ILC dans le contrôle de la réponse adaptative aux bactéries commensales in vivo. $A$. Les souris chimériques obtenues par le transfert des cellules $T$ et $B$ dans les souris congéniques Ragl ${ }^{-/-}$sont dépletées en ILC par l'anticorps anti-CD90.2. En l'absence des ILC, les souris développent des symptômes d'inflammation dus à la prolifération importante des $T C D 4^{+}$et à la production d'IgG spécifiques des bactéries commensales. Cette inflammation est corrigée par l'administration d'antibiotiques. B. Les souris congéniques naïves auxquelles on a administré des cellules T CD4 $4^{+}$spécifiques du peptide dérivé des bactéries commensales CBirl et des ILC préalablement chargées in vitro par ce peptide, ont un nombre diminué de cellules T CD4 ${ }^{+}$en prolifération et un taux d'IFN $\gamma$ (produit après immunisation) inférieur à celui de souris n'ayant pas reçu d'ILC. Les ILC sont capables de réguler la réponse T CD4. C. Les souris chez lesquelles on a éliminé l'expression du CMH-II dans les ILC ROR $\gamma \mathrm{t}^{+}$(CMH-IIILC) ont été obtenues par le croisement de souris exprimant le gène $\mathrm{H} 2-\mathrm{Abl}$ entouré de sites LoxP $\left(\mathrm{H}_{2}-\mathrm{Abl} \mathrm{I}^{f l}\right)$ avec des souris exprimant la recombinase Cre sous le contrôle du promoteur Rorc. En l'absence d'expression de CMH-II par les ILC, la réponse T et B dans ces souris est accrue, bien que le nombre des ILC ne change pas. $D$. Les souris $\mathrm{Ragl}^{-/-}$ayant reçu des lymphocytes T CD4 ${ }^{+}$de souris CMH-II ${ }^{\Delta L C}$ perdent du poids et développent des signes d'inflammation intestinale (prolap-

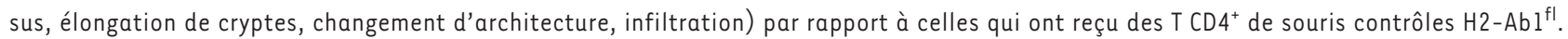
L'administration orale d'antibiotiques aux souris $\mathrm{CMH}-\mathrm{II}^{\Delta I L C}$ précédant le prélèvement des lymphocytes T CD4 ${ }^{+}$abroge la capacité qu'ont ces derniers à induire l'inflammation intestinale et la perte de poids.

Le rôle régulateur des ILC dans la réponse aux bactéries commensales a été confirmé dans le système de transfert de cellules $\mathrm{T} \mathrm{CD} 4^{+}$purifiées de souris $\mathrm{H2}-\mathrm{Abl}^{\mathrm{fl}}$ ou CMH-II ${ }^{\Delta l L C}$ à des souris Ragl ${ }^{-/-}$(Figure 3D). Ce transfert a provoqué l'inflammation intestinale et la perte de poids des animaux, confirmant qu'en absence d'ILC, les bactéries commensales entraînent le développement des réponses $\mathrm{TCD}^{+}$et le déclenchement d'une inflammation intestinale.

\section{Perspectives}

Il existe une certaine controverse par rapport au rôle des ILC chez l'homme. Des études antérieures ont montré que certaines populations d'ILC ROR $\gamma \mathrm{t}^{+} \mathrm{s}^{\prime} \mathrm{ac}-$ cumulent sélectivement dans le côlon 
des patients atteints de maladie de Crohn et y produisent des cytokines proinflammatoires pouvant déclencher une inflammation [10].

Contrairement à ces données, l'étude de Hepworth et al. chez la souris suggère que les ILC jouent un rôle régulateur de la réponse adaptative aux bactéries commensales [9]. L'absence d'expression de molécules de costimulation par les ILC de groupe 3 peut être un avantage pour restreindre la réponse de l'hôte aux bactéries commensales. II reste à déterminer si ce mécanisme est aussi impliqué dans le rétrocontrôle de la réponse adaptative chez l'homme. $\diamond$ Innate lymphoid cells control the intestinal commensal bacteria adaptive response

\section{LIENS D'INTÉRÊT}

L'auteur déclare n'avoir aucun lien d'intérêt concernant les données publiées dans cet article.

\section{RÉFÉRENCES}

1. Clemente JC, Ursell LK, Parfrey LW, Knight R. The impact of the gut microbiota on human health: an integrative view. Cell 2012; 148 : 1258-70.

2. Brenchley JM, Douek DC. Microbial translocation across the GI tract. Annu Rev Immunol 2012 ; 30 : 149-73.

3. Cherrier M. Les cellules lymphoïdes innées. Med Sci (Paris) $2014 ; 30: 280-8$

4. Spits H, Cupedo T. Innate lymphoid cells: emerging insights in development, lineage relationships, and function. Annu Rev Immunol $2012 ; 30$ : 647-75.

5. Spits H, Artis D, Colonna M, et al. Innate lymphoid cells: a proposal for uniform nomenclature. Nat Rev Immunol 2013; 13: 145-9.

6. Sonnenberg GF, Artis D. Innate lymphoid cell interactions with microbiota: implications for intestinal health and disease. Immunity 2012 ; 37 : 601-10
7. Walker JA, Barlow JL, McKenzie AN. Innate lymphoid cells: how did we miss them? Nat Rev Immunol 2013 13: $75-87$.

8. Sonnenberg GF, Monticelli LA, Alenghat T, et al. Innate lymphoid cells promote anatomical containment of lymphoid-resident commensal bacteria. Science 2012 ; 336 : 1321-5

9. Hepworth MR, Monticelli LA, Fung TC, et al. Innate lymphoid cells regulate $\mathrm{CD} 4+\mathrm{T}$-cell responses to intestinal commensal bacteria. Nature 2013 ; 498 : 113-7.

10. Geremia A, Arancibia-Carcamo CV, Fleming MP, et al. IL-23-responsive innate lymphoid cells are increased in inflammatory bowel disease. J Exp Med 2011 ; 208 . 1127-33.

11. El Kaoutari A, Armougom F, Raoult D, Henrissat B. Le microbiote intestinal et la digestion des polysaccharides. Med Sci (Paris) 2014 ; 30 : 259-65.

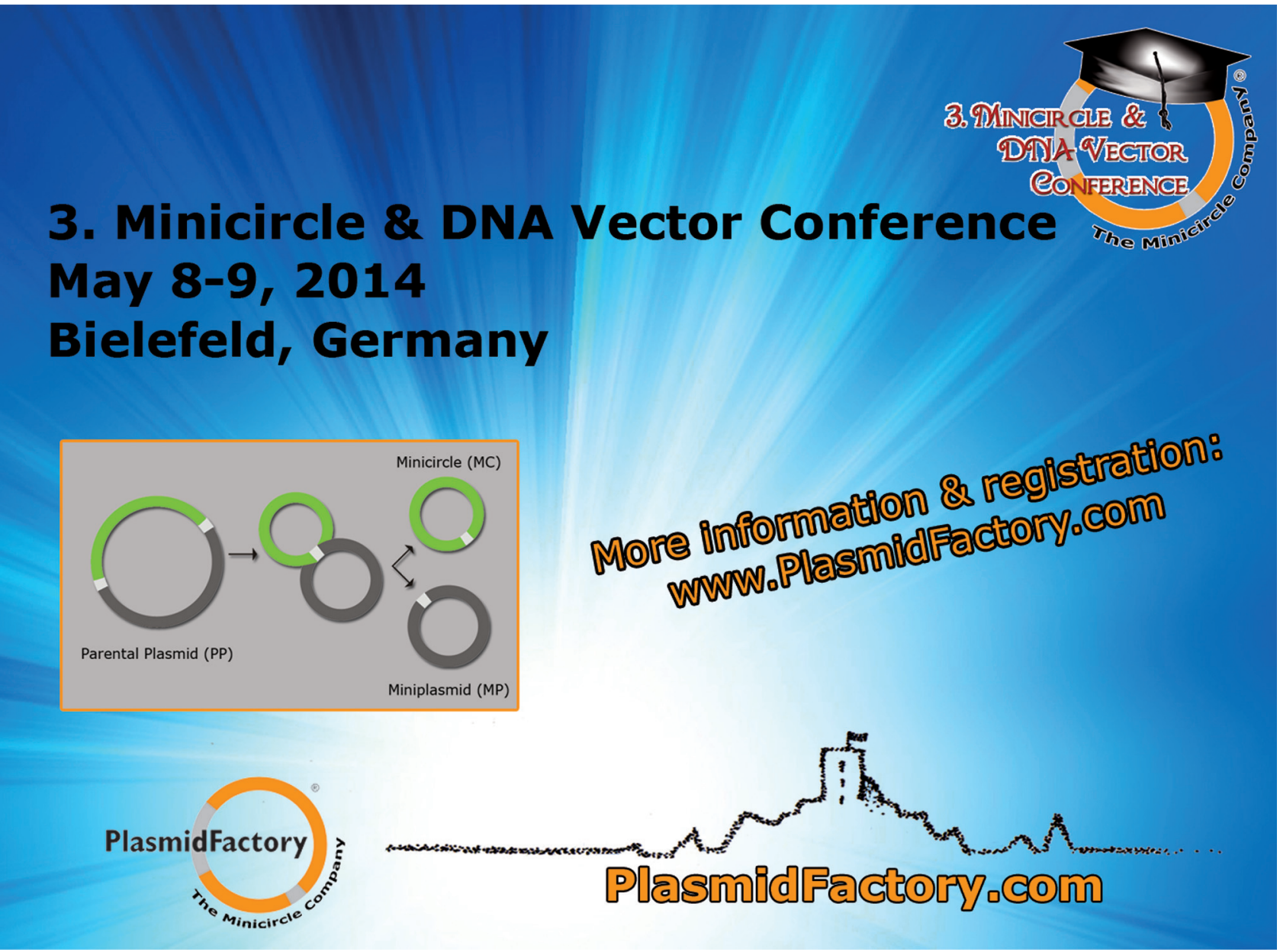

